\title{
Inbreeding reveals stronger net selection on Drosophila melanogaster males: implications for mutation load and the fitness of sexual females
}

\author{
MA Mallet and AK Chippindale \\ Department of Biology, Queen's University, Kingston, Ontario, Canada
}

\begin{abstract}
Stronger selection on males has the potential to lower the deleterious mutation load of females, reducing the cost of sex. However, few studies have directly quantified the strength of selection for both sexes. As the magnitude of inbreeding depression (ID) is related to the strength of selection, we measured the cost of inbreeding for both males and females in a laboratory population of Drosophila melanogaster. Using a novel technique for inbreeding, we found significant ID for both juvenile viability and adult fitness in both sexes. The genetic variation responsible for this depression in fitness appeared to be recessive for adult fitness $(h=0.11)$ and partially additive for juvenile viability $(h=0.29)$. ID was identical
\end{abstract}

across the sexes in terms of juvenile viability but was significantly more deleterious for males than females as adults, even though female X-chromosome homogamety should predispose them to a higher inbreeding load. We estimated the strength of selection on adult males to be 1.24 greater than on adult females, and this appears to be a consequence of selection arising from competition for mates. Combined with the generally positive intersexual genetic correlation for inbred lines, our results suggest that the mutation load of sexual females could be meaningfully reduced by stronger selection acting on males. Heredity (2011) 106, 994-1002; doi:10.1038/hdy.2010.148; published online 1 December 2010

Keywords: sexual selection; mutation load; adult fitness; viability; inbreeding; intersexual genetic correlation

\section{Introduction}

The power of sexual selection to shape traits directly related to reproduction is well established, but the consequences of sexual selection for the rest of the genome have only recently begun to emerge. The genic capture hypothesis suggests that mutations throughout the genome could be affected by sexual selection, as the overall health and vigor of the organism may alter the expression of traits preferred by females (Rowe and Houle, 1996; Tomkins et al., 2004). In addition, other sexually selected traits such as mate finding, coercion and endurance rivalry (Andersson and Iwasa, 1996) are likely candidates for condition dependence. At the same time, theory predicts that stronger sexual selection on males can lower the number of deleterious mutations affecting females at shared sexually selected loci (Agrawal, 2001; Siller, 2001). Taken together, these ideas imply that malebiased selection could be a force that improves mean female fitness on a genome-wide scale (Kodric-Brown and Brown, 1987; Whitlock and Agrawal, 2009). Under some conditions (for example, a diploid genomic mutation rate of 1 and selection on males being approximately twice as strong as selection on females), the mean fitness of females can be double that of females in asexual

Correspondence: MA Mallet, Department of Biology, Queen's University, Biosciences Complex, 116 Barrie Street, Kingston, Ontario, Canada K7L 3N6. E-mail:martin.mallet@queensu.ca

Received 19 May 2010; revised 7 September 2010; accepted 25 October 2010; published online 1 December 2010 populations (Agrawal, 2001; Siller, 2001), potentially accounting for the cost of sexual reproduction. Although populations enjoying increased female fitness through this mechanism may not be resistant to invasion by new asexual mutants, who in the short term benefit from both past bouts of selection and freedom from the cost of producing males (Salathé, 2006), reductions in mutation load will nevertheless have important consequences for populations. For example, reducing the number of segregating mutations will reduce inbreeding depression (ID) for female fitness: a substantial contributor to extinction risk (Frankham, 2005).

Whitlock and Agrawal (2009) have recently pointed out that measuring the total strength of selection on both sexes can be used to predict the effect of stronger selection on males for female mutation load, but they also highlight a lack of relevant empirical data. One approach to quantifying differences in net selection between the sexes is to independently quantify their response to inbreeding. Greater ID for one sex may be a fundamental feature of most sexual populations for at least three reasons. First, the heterogamety of sex chromosomes predisposes each sex toward different costs of inbreeding. In XY sex-determination systems, one expects greater ID for females than for males simply because the $X$ chromosome is always effectively homozygous for males. In addition, mutations on the $X$ with female-limited effects are not exposed to selection when expressed in males (Demuth and Wade, 2007); the reverse would be true in ZW systems. Second, the presence of widespread sexually antagonistic variation 
will result in sex-specific ID, as inbreeding would fix alleles having opposite fitness effects in each sex. Third, if one sex experiences stronger net selection, then the cost of inbreeding will be greater for that sex for a given mutation. By comparing the cost of inbreeding for the same genotype expressed in both males and females, we will gain insight into the intensity of selection upon each sex at the whole-genome scale.

Meaningful comparisons of ID between the sexes based on current data is difficult, however, in part because few researchers have explicitly set out to compare inbreeding load for males and females and ID is environment sensitive. The latter may make it dubious to compare inbreeding load from different studies, even within the same species. Where compared, however, the majority of results point to males as the more fragile sex. For example, in wild populations a higher sensitivity to inbreeding for males has been suggested for red deer (Slate et al., 2000), although in an isolated population of song sparrows ID for lifetime reproductive success was greater for females (Keller, 1998). In the laboratory, male flour beetles experienced stronger ID than females did (Pray and Goodnight, 1995), and male virility in Drosophila, an important component of male fitness, appears to be more strongly affected by inbreeding than female fertility (Brittnacher, 1981). Similarly, Miller and Hedrick (1993) made the second chromosome completely isogenic in Drosophila melanogaster and found that inbreeding had a stronger impact on male virility than on female fecundity. Meagher et al. (2000) showed that housing mice under competitive, semi-natural conditions increased the average magnitude of ID by 4.5 times compared with standard laboratory conditions. Most of this change came from a dramatic reduction in male reproductive success under semi-natural conditions, accounting for virtually all of the increase in average ID.

Although suggestive, these data are not sufficient to evaluate the hypothesis that females can benefit from stronger selection on males: it is also necessary to demonstrate that the alleles responsible for the inbreeding response of males have the same directional effect on females. Measurements of the strength of selection acting on each sex must, therefore, be supplemented by an estimate of the intersexual genetic correlation, because sex-limitation and sexually antagonistic alleles can cause net selection to be stronger for males with no benefit to females.

In order to obtain a meaningful estimate of selection on both sexes, as well as the intersexual genetic correlation for fitness, we must express the same genotypes in each sex and measure their fitness in a common environment. Establishing a controlled environment that is defined and reproducible, however, often comes at the cost of relevance to the natural environment to which the study organism is actually adapted, which in most cases is highly complex and variable. The use of laboratoryadapted populations that have been maintained under relatively constant conditions for hundreds of generations alleviates some of these concerns, because estimates of evolutionary parameters can be performed in the relevant selective environment.

We therefore created a set of inbred lines using a novel extension of the $D$. melanogaster clone-generator system developed by Rice and colleagues (Rice, 1996;
Chippindale et al., 2001). The approach, which we dubbed 'directed inbreeding' (DI), allowed us to make a set of genomic haplotypes extracted from a large laboratory-adapted population homozygous, and then express them in both sexes, in both the inbred and outbred state. We then characterized performance throughout the fly's life cycle, measuring both juvenile viability and adult reproductive success, under conditions to which the population had adapted for $\sim 800$ generations. This approach has some of the same limitations of traditional studies with balancer chromosomes, notably an averaging effect over many loci and resulting inability to resolve the effects of individual loci, but several advantages as well. Specifically, it allowed us to shed all artificial genetic aberrations and markers in the experimental generation, to recreate the ancestral competitive environment in almost every detail, to measure the fitness of each sex independently in the same experiment, as well as to estimate the intersexual genetic correlation for the genomes studied.

We show that the effects of inbreeding vary strongly by sex and life-history stage, further highlighting the importance of accounting for sex differences in studies of fitness and mutation load. Specifically, we confirm results from previous studies suggesting that there is a substantially higher cost of inbreeding for Drosophila males, we show that this additional cost is strongest in the adult stage, and demonstrate that male and female inbred fitness is largely, positively correlated. Taken together, these results satisfy the theoretical requirements for male-biased selection to cause a reduction in female mutation load.

\section{Materials and methods}

\section{Stocks and culture conditions}

The focal population was the Ives (IV) population of D. melanogaster. IV was isolated from a wild-caught sample of 200 males and 200 females in Amherst, Massachusetts (Rose and Charlesworth, 1981). At the time, the south Amherst population was thought to be continuous and overwintering, and had been monitored since at least 1931 (Ives, 1970). From 1981 onwards, the IV laboratory population has been maintained as a large outbred stock at a minimum population size of 1000 individuals at $25^{\circ} \mathrm{C}, 50 \%$ relative humidity, on a 14-day, discrete generation cycle with moderate densities of 60-120 individuals per $10 \mathrm{ml}$ of banana/agar/killed-yeast medium (Rose, 1984). On day 14/day 0, the population is placed under $\mathrm{CO}_{2}$ anesthesia, mixed and transferred to new vials to oviposit until $\sim 100$ eggs are laid in each vial. This usually takes $\sim 30 \mathrm{~min}$ and represents the only opportunity for offspring production.

In 2004, a replica of the IV population was created by backcrossing the IV population to a population bearing the recessive $b w^{1}$ (brown eyes) allele and denoted as $I V_{b w}$. The $\mathrm{IV}_{b w}$ population serves as an outbred, genetically similar population for use as competitors against IVderived individuals in measurements of fitness. This marker has few deleterious side effects and the $\mathrm{IV}_{b w}$ stock is vigorous. Periodically, the IVs are introgressed into the $\mathrm{IV}_{b w}$ to prevent drift between the focal (IV) and competitor $\left(\mathrm{IV}_{b w}\right)$ populations. 
Inbred lines

The 18 inbred lines used for this study were generated using a novel application of the clone-generator system of markers and chromosome rearrangements (Chippindale et al., 2001). A selection of haploid genomes derived from the IV population and known to possess significant genetic variation for fitness in each sex provided the raw material for the creation of the inbred lines. These genomic haplotypes were made homozygous by mating males bearing the haplotype of interest in the heterozygous state along with a marked translocation (T(2:3)rdgc st in ri $\left.p^{P} b w\right)$ to wild-type females, discarding the marked translocated autosomes in the female progeny. These females, having received a genomic haplotype from their father, were collected as virgins and again crossed to males with the haplotype of interest, along with the marked translocation, for 10 generations (Figure 1). With each successive cross, the proportion of genes identical to the founding line's haplotype increases by $50 \%$. Subsequently, these lines were maintained at population sizes of 10 females and 6 males to minimize genetic variation. A reduced sex ratio was employed to reduce mate harm to females, minimizing the effective population size while maintaining productivity. Periodically, they were backcrossed again to their founding lines. As the final result of these crosses is the homozygous version of a specific genomic haplotype, we named this method directed inbreeding (DI).

The DI method has several advantages over traditional inbreeding methods, such as brother-sister mating. First, DI is less susceptible to the stochastic loss of mutations resulting from the inbreeding process. Brother-sister mating exposes mutations to both drift and selection during the inbreeding process, which could result in
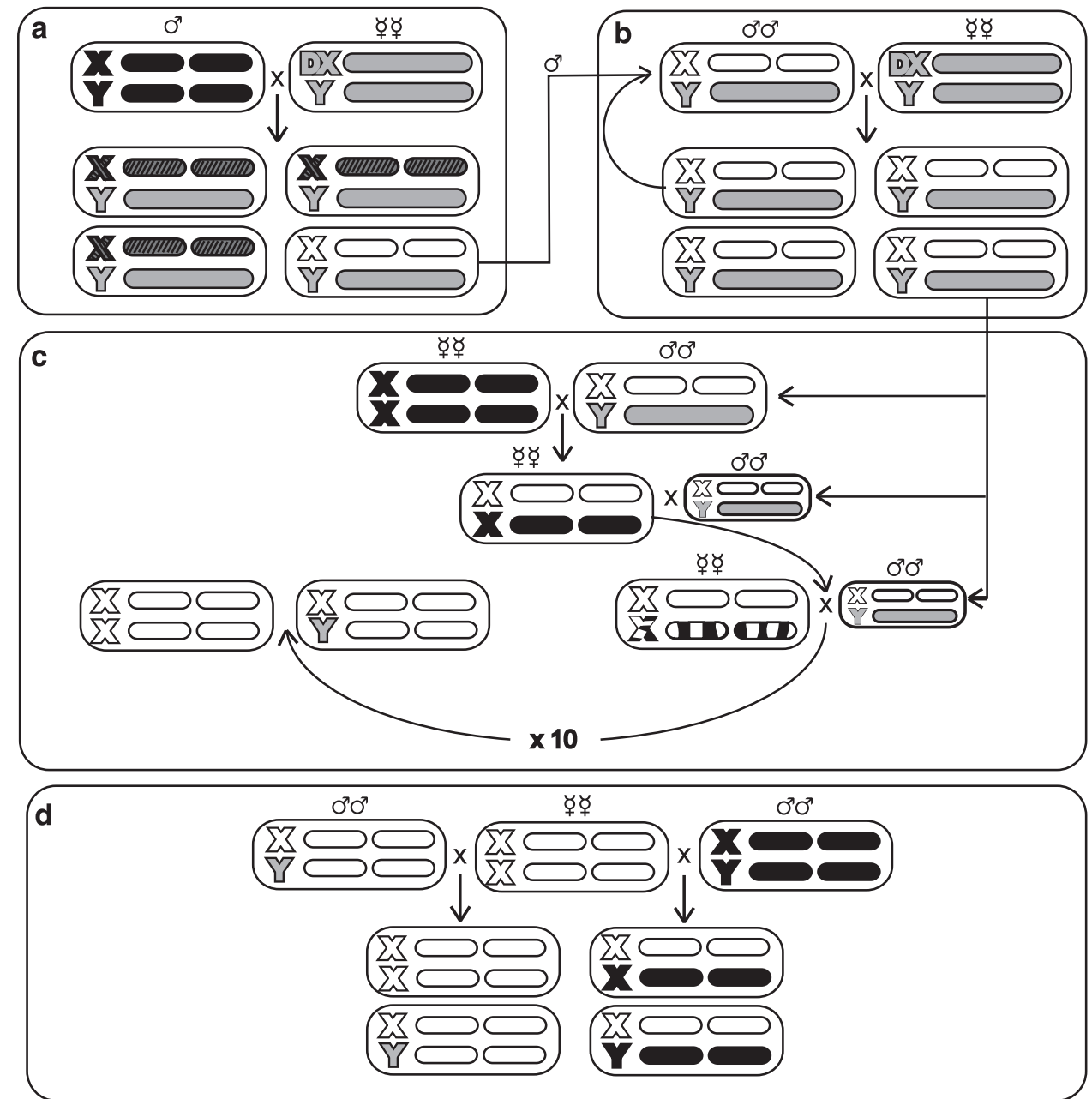

Figure 1 Breeding schemes used in this experiment. (a) Generation of hemiclone lines. A single male from the IV population possessing an unknown genotype (black) was crossed to virgin clone-generator females possessing a compound X (C(1)DX, $y$, f, depicted by DX symbol), a Y chromosome and a marked translocation of chromosomes II and III (T(2:3)rdgc st in ri $p^{P} b w$, depicted by solid bar spanning the two major autosomes because these chromosomes cosegregate in surviving offspring). The resulting male offspring possesses one of four possible genotypes, consisting of a set of X plus wild-type autosomes inherited paternally, with a $\mathrm{Y}$ and marked translocation inherited maternally. (b) A single male is then randomly selected to again cross to clone-generator females, thus fixing a genomic paternal haplotype (white) within a line. The resulting hemiclone line is propagated by crossing males heterozygous for the marked translocation with clone-generator females. (c) DI. Males from a given hemiclone line are first crossed to virgin wild-type IV females with an unknown genotype (black). Virgin females are collected from this cross, which are now heterozygous for the founding hemiclonal haplotype. These females are again crossed to hemiclone males, and the process is repeated 10 times to yield lines inbred for the founding haplotype. (d) Generation of experimental flies. Inbred females from a given line are crossed to either inbred males (white) or outbred males (black) to yield outbred and inbred flies of both sexes. 
genomes purged of a fraction of their deleterious mutation load. DI reintroduces all of the mutations present in the founding genome in each generation of inbreeding, preventing the loss of deleterious mutations. The exception, common to all inbreeding approaches, is that one cannot fix sterile or lethal mutations within a line; these are thought to contribute to approximately half of the total mutation load in D. melanogaster (Lynch and Walsh, 1997). Second, the DI approach has the advantage of capturing all three of D. melanogaster's major chromosomes in the same experiment. With this species, recombination-suppressing balancer chromosomes are restricted to capturing and manipulating at most two of the major chromosomes simultaneously. Manipulating the whole genome should give a more accurate picture of the population consequences of genome-wide processes, while leaving open the potential for later deconstruction and analysis at the chromosomal level using balancer techniques.

Each inbred line was used to experimentally generate both inbred and outbred flies for use in measurements of fitness (Figure 1). Outbred individuals were created by crossing females from the line of interest with randomly selected wild-type males from the IV population. Inbred individuals were created by crossing these females with males from the inbred line. In this manner, both outbred and inbred individuals have inbred mothers with the same genotype, eliminating differences between outbred and inbred experimental flies because of maternal effects. Although the potential for ID exists along the entire genome for females, it was restricted to the autosomes for males, as both outbred and inbred males of a given line express the same $\mathrm{X}$ chromosome hemizygously.

\section{Viability assay}

Juvenile viability was assessed by placing 50 eggs from a given genotype in a vial along with 50 eggs from a standard competitor $\left(\mathrm{IV}_{b w}\right)$, mimicking standard culture densities. After 12 days, sufficient for virtually all adults to emerge (confirmed by visual inspection), egg-to-adult viability was assessed by counting and scoring progeny for both sex and eye color. Each genotype/treatment combination was replicated five times.

\section{Adult fitness assay}

Inbred or outbred individuals of the sex/genotype of interest were collected during peak emergence (day 9 from date of oviposition) and singly transferred to a vial containing an age-synchronized culture of $\mathrm{IV}_{b w}$ that was reared at standard culture densities ( $\sim 100$ eggs/vial). After 5 days (day 14), experimental vials were anaes- thetized with $\mathrm{CO}_{2}$ for $2.5 \mathrm{~min}$ and transferred to fresh vials to allow for oviposition $(30 \mathrm{~min})$, mimicking standard culture conditions. The adults were then removed from the vials and the sex/number of progeny from the target individuals (distinguishable by their red eyes) was scored 12-14 days later. Each genotype/ treatment/sex combination was replicated 30 times.

\section{Results}

\section{Sex-specific ID in the IV population}

For both juvenile and adult life-history stages, inbreeding caused a measurable depression in fitness. Based on analysis of line means, mean viability in the outbred treatment was $61.2 \%$ for males $(95 \% \mathrm{CI}=(55.1,67.3))$ and $61.7 \%$ for females $(95 \% \mathrm{CI}=(57.0,66.3 \%))$, while it was $78.9 \%$ for the $\mathrm{IV}_{b w}$ competitors (male $95 \%$ $\mathrm{CI}=(78.0,82.4)$, female $95 \% \mathrm{CI}=(74.9,79.5))$. The lower viability of outbred IV individuals, relative to the $\mathrm{IV}_{b w}$ competitors, is likely the result of maternal effects from their inbred mothers. ID for juvenile viability was $35 \%$ for both sexes, with inbred male viability at $40.3 \%$ $(95 \% \mathrm{CI}=(32.8,47.9))$ and female viability at $40.0 \%(95 \%$ $\mathrm{CI}=(31.3,48.7))$. The total number of $\mathrm{IV}_{b w}$ competitors in the vials containing inbred flies was unchanged (one-way ANOVA, $P$-value $=0.25$ ), suggesting that the reduction in viability in the inbred lines was not because of competitive exclusion by $\mathrm{IV}_{b w}$ individuals. Indeed, no correlation was found between the number of red-eyed progeny and the number of brown-eyed progeny within a vial $\left(P\right.$-value $=0.55, r^{2}=0.002$, slope $\left.=0.124\right)$. A threefactor ANOVA was performed on the viability data (using sex and degree of inbreeding as fixed effects, and line as a random effect), which shows significant genetic variation for viability and a significant effect of inbreeding in the IV population but no sex $\times$ inbreeding interaction, despite the potential for females to express ID on the $X$ chromosome (Table 1 ).

For adult fitness, males and females differed in both their outbred and inbred mean fitness. Based on analysis of line means, males had higher outbred fitness (male mean $=2.69,95 \% \mathrm{CI}=(2.09,3.28)$, female mean $=$ $1.99,95 \% \mathrm{CI}=(2.25,1.72))$ but lower inbred fitness (male mean $=0.267,95 \% \mathrm{CI}=(0.152,0.382)$, female mean $=$ $0.719,95 \% \mathrm{CI}=(0.546,0.892))$. The higher mean for outbred males could be because of an advantage of red eyes in mate competition against $b w$-bearing males. ID for adult fitness was, therefore, $90.1 \%$ for males and $63.9 \%$ for females, respectively. As male and female outbred means were different and ID is a relative measure, male and female adult fitness was divided by

Table 1 Mixed-effects ANOVA on juvenile viability

\begin{tabular}{|c|c|c|c|c|}
\hline Source & Mean square numerator & Numerator degrees of freedom & F-ratio & P-value \\
\hline Sex & 0.0261 & 1 & 0.0016 & 0.97 \\
\hline Line & 141.02 & 16 & 2.48 & 0.043 \\
\hline Treatment & 2363.5 & 1 & 43.05 & $<0.0001$ \\
\hline Sex $\times$ line & 15.851 & 16 & 1.14 & 0.40 \\
\hline Sex $\times$ treatment & 0.652 & 1 & 0.047 & 0.83 \\
\hline Line $\times$ treatment & 54.96 & 16 & 3.94 & 0.0046 \\
\hline Sex $\times$ line $\times$ treatment & 13.94 & 16 & 1.17 & 0.29 \\
\hline
\end{tabular}

All sources of variance that included line were treated as random-effects terms. 
Table 2 Mixed-effects ANOVA on adult fitness

\begin{tabular}{|c|c|c|c|c|}
\hline Source & Mean square numerator & Numerator degrees of freedom & F-ratio & P-value \\
\hline Sex & 6.51 & 1 & 3.85 & 0.065 \\
\hline Line & 17.82 & 17 & 1.49 & 0.29 \\
\hline Treatment & 1479.9 & 1 & 228.34 & $<0.0001$ \\
\hline Sex $\times$ line & 14.01 & 17 & 1.56 & 0.18 \\
\hline Sex $\times$ treatment & 143.1 & 1 & 5.10 & 0.036 \\
\hline Line $\times$ treatment & 8.41 & 17 & 0.81 & 0.66 \\
\hline Sex $\times$ line $\times$ treatment & 9.92 & 17 & 2.85 & $<0.0001$ \\
\hline
\end{tabular}

All sources of variance that included line were treated as random-effects terms.

their outbred group means before testing for sex-specific declines in fitness because of inbreeding by a three-way ANOVA, which showed a significant sex $\times$ inbreeding interaction (Table 2). The three-way interaction between clone, sex and degree of inbreeding was also significant, and this is because of a significant clone $\times$ inbreeding interaction for males, but not for females. We also directly tested for sex-specific ID in adult fitness by creating 5000 bootstrap replicates of the ratio of inbred to outbred fitness for each sex, using line means. This gave us an estimate of $90.1 \%(95 \% \mathrm{CI}=(0.865,0.933))$ for male ID, $64.0 \%(95 \% \mathrm{CI}=(0.586,0.689))$ for female ID. Males thus experienced 1.41 times more ID than females $(95 \%$ $C I=(1.30,1.56))$. The higher cost of inbreeding to adult male fitness was observed despite males having a smaller genomic target for ID, and despite the expectation that inbred experimental females would likely suffer an additional reduction in fitness because of reduced viability in their progeny compared with the offspring of outbred experimental females.

\section{Inbreeding load and dominance coefficient}

The inbreeding load and average degree of dominance for mutations segregating in our experiment were estimated separately for each life-history stage and sex, using line means (Table 3). Many experimental designs involving inbred lines create outbred individuals by crossing two inbred lines and can estimate the average dominance coefficient by regressing outbred fitness on the sum of the parental inbred fitness values (for example, Willis, 1999). As we created outbred flies by expressing each focal genome against a collection of random wild-type backgrounds, we instead used the estimator $A /\left(2(A+B)\right.$ ) (where $A$ is $-\ln \left(\mathrm{W}_{\mathrm{OUT}}\right), B$ is $-\ln \left(\mathrm{W}_{\mathrm{IN}} / \mathrm{W}_{\mathrm{OUT}}\right)$, and the reported values are based on the average across lines). This provides a reasonable estimate of the arithmetic mean dominance for segregating alleles (Lynch and Walsh, 1997 but see ; Fernández et al., 2004). Estimates for dominance were very similar between the sexes, with genetic variation affecting adult fitness being more recessive than variation with deleterious effects for juvenile viability. These results are similar to those of Mackay (1985), who found evidence for additivity for viability but an overall dominance coefficient for net fitness of 0.13 in a different population of Drosophila.

\section{Variance for fitness across sexes and life stages}

We tested for significant additive genetic variance among genomes in the IV population by fitting a random-effects ANOVA for each sex/developmental stage/inbreeding level combination, using line as the only factor.
Table 3 Dominance and load estimates for both sexes and developmental stages

\begin{tabular}{lccc}
\hline & B & A + B & h \\
\hline Male viability & 0.48 & 0.98 & 0.29 \\
Female viability & 0.54 & 1.03 & 0.29 \\
Male adult fitness & 2.56 & 3.23 & 0.10 \\
Female adult fitness & 1.12 & 1.51 & 0.12 \\
\hline
\end{tabular}

$B$ is $-\ln \left(\mathrm{W}_{\mathrm{IN}} / \mathrm{W}_{\text {OUT }}\right)$ and $A$ is $-\ln \left(\mathrm{W}_{\text {OUT }}\right) . B$ is a measure of inbreeding load and the number of lethal equivalents in a genome is bounded by $B$ and $A+B$. To calculate $A$ for adult fitness, $W_{\text {OUT }}$ and $\mathrm{W}_{\text {IN }}$ were calculated relative to the highest outbred line mean.

Table 4 Coefficients of variation for fitness components

\begin{tabular}{lcccr}
\hline & $C V_{A}$ & $C V_{E}$ & $C V_{P}$ & P-value \\
\hline Viability & & & & \\
$\quad$ Outbred males & 0.15 & 1.51 & 1.52 & 0.0020 \\
$\quad$ Inbred males & 0.34 & 2.24 & 2.26 & $<0.0001$ \\
Outbred females & 0.10 & 1.32 & 1.32 & 0.0557 \\
$\quad$ Inbred females & 0.41 & 2.44 & 2.47 & $<0.0001$ \\
& & & & \\
Adult fitness & & & & \\
$\quad$ Outbred males & 0.38 & 1.07 & 1.13 & $<0.0001$ \\
$\quad$ Inbred males & 0.65 & 2.89 & 2.96 & 0.0015 \\
$\quad$ Outbred females & 0.20 & 0.79 & 0.81 & $<0.0001$ \\
$\quad$ Inbred females & 0.39 & 1.39 & 1.44 & $<0.0001$ \\
\hline
\end{tabular}

A significant effect of line was found for every treatment combination, except for female outbred viability (Table 4). For viability, the coefficient of phenotypic variation $\left(C_{P}\right)$ was corrected for the number of flies placed in each vial, and the coefficient of environmental variation $\left(\mathrm{CV}_{\mathrm{E}}\right)$ was estimated from the residual variance $\left(V_{P}-V_{A}\right)$. In all cases, the coefficient of additive genetic variation $\left(\mathrm{CV}_{\mathrm{A}}\right)$ increased with inbreeding, consistent with the exposure of recessive variation by inbreeding. Male $C V_{A}$ was generally higher than female $C_{\mathrm{A}}$, except for inbred juvenile viability. Residual variation $\left(\mathrm{CV}_{\mathrm{E}}\right)$ also increased with inbreeding, suggesting a higher susceptibility of inbred flies to environmental effects.

\section{Intrasexual correlations for juvenile viability and adult fitness}

Inbred line means tended to be correlated with outbred line means. We found a significantly positive correlation for outbred viability regressed on inbred viability for female line means $\left(P=0.016, r^{2}=0.33\right.$, slope $\left.=0.31\right)$, but no significant relationship between mean outbred and inbred male line means $\left(P=0.23, r^{2}=0.095\right.$, slope $\left.=0.25\right)$. For adult fitness, mean inbred fitness was significantly 

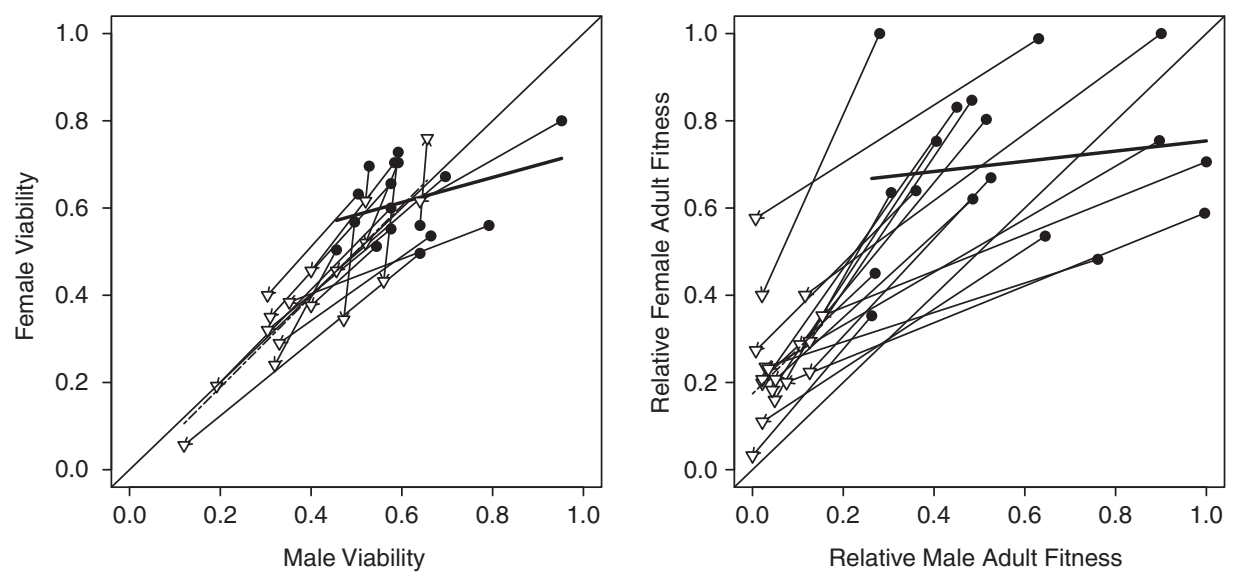

Figure 2 Intersexual correlations for juvenile viability and adult fitness under inbred and outbred experimental treatments. Outbred line means (solid circles) are connected to their corresponding fitness value as inbred lines (white triangles) by arrows. Solid regression lines represent the slope of the intersexual correlation for outbred lines and dashed regression lines show the slope of the intersexual correlation for inbred lines. The slope shown for inbred adult fitness was determined after removal of an outlier (see text). The 1:1 line is shown as a thin diagonal line spanning the whole plot.

correlated with outbred fitness for both sexes (males: $P=0.0495, r^{2}=0.22$, slope $=2.43$; females: $P<0.0001$, $r^{2}=0.62$, slope $=1.21$ ).

\section{Intersexual correlations for juvenile viability and adult fitness}

For both juvenile viability and adult fitness, inbreeding was associated with an increase in the intersexual correlation (Figure 2). For both developmental stages, there was no significant correlation between outbred male and female fitness values (viability: $P=0.14$, $r^{2}=0.14$, slope $=0.29$; adult fitness: $P=0.54, r^{2}=0.024$, slope $=0.12$ ). For the inbred lines, there was a highly significant positive intersexual relationship for juvenile viability $\left(P<0.0001, r^{2}=0.82\right.$, slope $\left.=1.04\right)$, but not for adult fitness $\left(P=0.39, r^{2}=0.045\right.$, slope $\left.=0.54\right)$. The lack of significant intersexual correlation for inbred adults was because of a single inbred line, which had both the highest female fitness and one of the lowest male fitness estimates $\left(\mathrm{W}_{\mathrm{IN}} / \mathrm{W}_{\mathrm{OUT}}=0.01\right.$ for males, $\mathrm{W}_{\mathrm{IN}} /$ $\mathrm{W}_{\text {OUT }}=0.58$ for females). Its status as a statistical outlier was confirmed using the Mahalanobis Distance (3.08), Jacknife Distance (4.96) and the $\mathrm{T}^{2}(9.51)$ criteria, and removing this line from the analysis results in a significantly positive and nearly isometric intersexual relationship for inbred adult fitness $\left(P=0.032, r^{2}=0.27\right.$, slope $=1.03$ ).

\section{Life-history correlations}

Within each sex, we found no relationship between line means for viability and adult fitness for either outbred (males: $P=0.21, r^{2}=0.10$, slope $=0.67$; females: $P=0.49$, $r^{2}=0.032$, slope $=0.34$ ) or inbred (males: $P=0.89$, $r^{2}=0.0014$, slope $=0.012$; females: $P=0.482, r^{2}=0.034$, slope $=0.13)$ flies, indicating no detectable tradeoffs or pleiotropy between juvenile and adult performance in terms of whole-genome effects.

\section{Discussion}

We present the first measurements of the cost of inbreeding to both sexes across the entire genome and life history for a population of D. melanogaster, extending the findings of previous studies having only examined fitness proxies, a fraction of the genome, a limited portion of the life cycle, or a single sex. Although we found that both sexes suffered equal reductions in juvenile viability, males experienced much stronger declines in adult fitness, resulting in a substantially greater overall cost of inbreeding for males than for females. As the coefficient dominance was the same between the sexes, we attribute greater ID in males to stronger selection. Importantly, we recorded positive intersexual correlations for inbred fitness, indicating that selection against segregating mutations generally operates in the same direction for both sexes. Our data thus provide the first direct empirical support for the hypothesis that stronger selection on males has the potential to benefit females by reducing the number of deleterious mutations segregating in sexual populations.

\section{Comparison to previous studies}

The magnitude of ID in populations is relevant to many areas of evolutionary biology, and has been the subject of considerable study. Although remarkably few studies have explicitly set out to examine the sex specificity of ID, there are many individual estimates of ID in Drosophila for the fitness components reported here. Lynch and Walsh (1997) summarized the results for several studies measuring ID in D. melanogaster. Although the estimates were variable, mean ID for eggto-adult viability was, with complete inbreeding, 0.36 $(n=3)$ for the studies having employed D. melanogaster; almost identical to the ID of 0.35 reported here. Estimates of adult fitness were also quite variable, and no study simultaneously measured both male and female major fitness components. Mean ID for female fertility $(n=3)$ was 0.45 (0.64 in this study), and mean ID for male mating ability $(n=3)$ was 0.73 ( 0.90 in this study), which is in good qualitative agreement with our results. These similarities are especially striking in light of the differences in environments and populations between all studies. 
Estimating the strength of selection on the sexes for individual loci

Previous studies have shown that sexual selection can augment purifying selection for single mutations, but have generally been unable to directly compare the total strength of selection acting on males and females (reviewed by Whitlock and Agrawal, 2009). Genomelevel measures of sex-specific selection help to fill this critical gap, but it is nonetheless desirable to relate genome-wide measurements to the processes operating at individual loci. Most models attempt to capture the effects of sexual selection on the fitness consequences of segregating mutations (expressed as mutation load) by considering the relative strength of selection on males versus females $(\alpha)$ at individual loci, given by

$$
\alpha=\frac{s_{\mathrm{m}}}{s_{\mathrm{f}}}
$$

When an individual is homozygous for a single mutation, the fitness of its genotype in males and females can be expressed as

$$
\begin{aligned}
& w_{\mathrm{f}}=1-s_{\mathrm{f}} \\
& w_{\mathrm{f}}=1-\alpha \times s_{\mathrm{f}}
\end{aligned}
$$

And we can directly calculate

$$
\alpha=\frac{1-w_{\mathrm{m}}}{1-w_{\mathrm{f}}}
$$

For a given inbred line, which might contain many mutations, and for which we have fitness data for both sexes, we can calculate in a similar fashion:

$$
A=\frac{1-W_{\mathrm{m}}}{1-W_{\mathrm{f}}}
$$

If the fitness effects of mutations are multiplicative (that is, no epistasis) we will have:

$$
\begin{aligned}
& W_{\mathrm{f}}=\left(w_{\mathrm{f}}\right)^{\mathrm{n}} \\
& W_{\mathrm{m}}=\left(w_{\mathrm{m}}\right)^{\mathrm{n}}
\end{aligned}
$$

where $n$ is the number of mutations. If $s_{\mathrm{f}}$ and $\alpha$ are variable across loci, we can replace $w_{\mathrm{m}}$ and $w_{\mathrm{f}}$ by their geometric means $\left(\bar{w}_{\mathrm{m}}\right.$ and $\left.\bar{w}_{\mathrm{f}}\right)$, and define:

$$
\begin{aligned}
\hat{s}_{\mathrm{f}} & =1-\bar{w}_{\mathrm{f}} \\
\hat{s}_{\mathrm{m}} & =1-\bar{w}_{\mathrm{m}} \\
\hat{\alpha} & =\hat{s}_{\mathrm{m}} \\
\hat{s}_{\mathrm{f}} &
\end{aligned}
$$

Substituting these values yields

$$
A=\frac{1-\left(1-\hat{\alpha} \times \hat{s}_{\mathrm{f}}\right)^{n}}{1-\left(1-\hat{s}_{\mathrm{f}}\right)^{n}}
$$

It is apparent that $A=\hat{\alpha}$ only when $n=1$, and $A$ tends toward 1 as the number of mutations in a given genome increases. Thus, $A$ underestimates $\hat{\alpha}$ when $\hat{\alpha}>1$ (this study), and would overestimate it if $\hat{\alpha}$ were smaller than one. For this reason, measurements of sex-specific selection at the genome level are likely to underestimate the strength of selection at individual loci, and $A$ can be considered equivalent to $\hat{\alpha}_{\min }$.

\section{Stage- and sex-specificity of ID}

We found that females did not suffer a greater cost of inbreeding at the juvenile stage $(A=1.02)$, as measured by egg-to-adult viability. This result is perhaps surprising, because of the expected additional cost of inbreeding to females resulting from the $X$ chromosome ( $20 \%$ of the total gene content). Combined with the strongly positive intersexual correlation for juvenile viability, this result suggests that the $X$ chromosome harbors few deleterious mutations affecting viability in the IV population. This finding is consistent with an earlier study of a different, wild population of $D$. melanogaster, which found low X-linked viability load along with a positive intersexual correlation for the variation in viability on the X (Eanes et al., 1985). We speculate that viability selection on males, where the $X$ is expressed hemizygously, has reduced the inbreeding load for females, thus suggesting a specific instance in which natural selection on males has resulted in fewer mutations segregating in the population, with viability benefits for females.

For adult fitness, the magnitude of ID was much higher for males than for females. A single line was exceptional in that the cost of inbreeding was extremely high for males ( $A$ for this line was 2.34, 3.5 s.d. away from the group mean), and was identified as a bivariate outlier in the inbred group (see Results) because of its combination of unusually high inbred female fitness and low inbred male fitness. For any given individual genome, a stronger decline in relative fitness in males could be because of the presence of sexually antagonistic alleles, the presence of alleles with sex-limited effects on fitness, or to a higher coefficient of selection on shared alleles. The relative importance of these three modes of allelic effect across the genome will determine the general magnitude and sign of the intersexual genetic correlation for fitness. Without detailed genetic and phenotypic characterization it is unfortunately impossible to speculate on the reason for a specific line's departure from the group response, but for the remaining 17 lines, the intersexual genetic correlation $\left(\mathrm{r}_{\mathrm{MF}}\right)$ in inbred flies was estimated to be 0.52 , with a slope of nearly 1 and net selection on males being greater than on females $(A=1.24)$. As we observed very little adult mortality, the observation of stronger net selection acting upon male fitness was most likely the result of reduced mating or post-copulatory fertilization success. We, therefore, attribute greater ID for males to stronger selection on the characters underlying success in sexual selection.

Our estimate of $A$ applies to autosomal mutations affecting adult fitness, because the $X$ chromosome does not contribute to ID in males. X-linked mutations affecting adult female fitness will cause our measure of $A$ for autosomal loci to be conservative, as they will increase the effect of inbreeding to females beyond the cost of autosomal loci, minimizing the difference between the sexes. Substantial X-linked ID for adult female fitness has previously been reported in another population of D. melanogaster (Wilton and Sved, 1979). The value of $A$ may also be underestimated if the offspring of inbred experimental females suffer reduced viability relative to the offspring of outbred experimental females as a result of maternal effects.

The generally positive genetic correlation we observed would seem to preclude the presence of large amounts of segregating sexually antagonistic variation in the IV population, or a dominant role for alleles with 
sex-limited effects. These results differ notably from studies by Chippindale et al. (2001) and from Innocenti and Morrow (2010), using the $\mathrm{LH}_{\mathrm{M}}$ population of $D$. melanogaster, who found a negative intersexual correlation for fitness (for example, $\mathrm{r}_{\mathrm{MF}}=-0.52$, from Innocenti and Morrow (2010)). The extent of intralocus sexual conflict may, therefore, vary considerably between populations, and the very high levels of sexually antagonistic variation segregating in the $\mathrm{LH}_{\mathrm{M}}$ population may not be a general feature of $D$. melanogaster populations. Nevertheless, the intersexual genetic correlation did appear weaker for adult fitness than for juvenile survival, which may indicate that sex-limited or sexually antagonistic alleles segregate in the IV population.

\section{Inbreeding load across life history}

We did not detect any correlation between juvenile and adult fitness, implying that benefits accrued by females from selection in adult males would not manifest themselves as viability gains in the offspring. Thus, the strength of selection on adult relative to juvenile fitness will determine the potential for stronger selection on adult males to affect the net fitness of females. In our sample of genomic haplotypes, inbreeding load was much higher for adult fitness than for juvenile fitness for both sexes. For females, adult load represented $67 \%$ of the total inbreeding load, whereas for males it was $84 \%$, implying stronger net selection overall for adults, and particularly for males. This stronger net selection may reflect a direct increase in the strength of selection active on adult reproductive success relative to juvenile viability (adult mortality is very low in the IV population), or may be caused by a larger number of adultspecific segregating mutations. Very few transcripts in D. melanogaster appear to be adult specific (Arbeitman et al., 2002), and most genes in this organism appear to be developmentally regulated (Stolc et al., 2004), which suggests that for the majority of genes the opportunity for selection exists across the entire life history. As the total inbreeding load appears to be dominated by the reproductive success of adults, we expect that a reduction in the numbers of mutations affecting adult female fitness as a result of sexual selection would also result in a meaningful increase in their mean fitness.

The potential for sexual selection to reduce total genetic load is likely to be taxon specific, as it depends on the relative strength of selection on adult versus juvenile fitness components as well as on the correlation between the two. There has been considerable interest in measuring the relative strength of selection across lifehistory stages; for a review see Hoekstra et al. (2001), who suggests that for many populations the strength of sexual selection, broadly defined, is generally greater than that of natural selection.

\section{Predicted benefits of sexual selection to adult female fitness}

The results in Agrawal (2001) assume that mutations act additively within a locus and that the sexual populations are completely outbred, but most mutations are recessive, and moderate levels of inbreeding are the norm for sexual populations. As both recessivity and population structure can separately yield benefits to sexual reproduction, the nature of which depends on the mutation rate (Agrawal and Chasnov, 2001), estimates of all these parameters are necessary to make quantitative predictions of benefits to females arising from sexual selection. To our knowledge, however, there have been no attempts to incorporate sexual selection, population structure, the dominance of mutations and mutation rate in one analysis. We provide a simple program to carry out and plot numerical simulations while varying all these parameters using $R$, a free statistical package ( $R$ Development Core Team, 2010; Supplementary Information).

Using a recent estimate of the diploid genomic deleterious mutation rate in Drosophila $(U=1.2$, Haag-Liautard et al., 2007) as a proxy for the mutation rate in the IV population, the average dominance coefficient for segregating mutations affecting adult fitness obtained in this study $(h=0.11)$, and our estimate of $\hat{\alpha}_{\min }(1.24)$, we speculate on the potential gain in adult female mean fitness for differing levels of population structure relative to a sexual population experiencing no sexual selection (Supplementary Figure S1). With no population structure $(f=0)$, equilibrium mean fitness is predicted to be $29 \%$ higher than the equivalent sexual population experiencing no additional selection on males and is $14 \%$ higher with complete inbreeding $(f=1)$. We also provide a contour plot of mean female fitness for a variety of combinations of mutation rate and $\hat{\alpha}$ at a moderate level of inbreeding $(f=0.2)$, which shows the potential for substantial gains in fitness from sexual selection, especially at high mutation rates (Supplementary Figure S2). Lower mutation loads in sexual populations could contribute to a lower extinction risk (Frankham, 2005). Stronger selection on males could be a reason for male-biased dispersal (Pusey, 1987) and may also have implications for breeding schemes aimed at improving fitness in captive populations (Wedekind, 2002).

\section{Future directions}

In addition to estimates of net selection on males and females for standing genetic variation (this study), estimates of the relative strength of selection for de novo mutations would be desirable. Segregating genetic variation may not reflect the underlying distribution of mutations and is expected to be enriched in certain classes of variation, including sexually antagonistic variation (Rice, 1984) and various forms of balancing selection (Peng et al., 1991). Other classes of mutation will be underrepresented and can only be studied with the input of new genetic variation. For example, we detected no ID for $X$-linked mutations affecting juvenile viability, presumably because of a lack of segregating variation. Although we inferred that strong viability selection on hemizygous $X$ chromosomes was the probable cause, a direct measure would be preferable. Deleterious mutations experiencing strong sexual selection in males may be rapidly purged, and estimates of the relative strength of selection in males and females might, therefore, be higher for new mutations than for segregating variation. We view estimates of sex-specific selection on segregating and de novo mutations as complementary, and are currently engaged in a mutation-accumulation experiment to address some of these outstanding questions.

Theoretical models for the benefit of stronger selection on males to sexual population have been presented (Agrawal, 2001; Siller, 2001; Whitlock and Agrawal, 
2009), and our work suggests further avenues for both empirical and theoretical work. Stronger selection on males will further amplify the benefits of mild inbreeding in purging deleterious mutations from sexual populations; we provide numerical estimates here but analytical predictions await a theoretical treatment. The potential for selection on males to reduce the deleterious mutation load affecting females is likely be even greater than current models predict.

\section{Conflict of interest}

The authors declare no conflict of interest.

\section{Acknowledgements}

This work was supported by a Discovery Grant from NSERC (Canada) to AKC, and by a CGS-D scholarship to MAM. We thank members of the Chippindale Lab, especially S Feagan, for help with data collection. We are also grateful to $\mathrm{H}$ Rundle for comments on an earlier draft of the manuscript as well as to A Agrawal and two anonymous reviewers for their suggestions.

\section{References}

Agrawal AF (2001). Sexual selection and the maintenance of sexual reproduction. Nature 411: 692-695.

Agrawal AF, Chasnov JR (2001). Recessive mutations and the maintenance of sex in structured populations. Genetics 158: 913.

Andersson M, Iwasa Y (1996). Sexual selection. Trends Ecol Evol 11: 53-58.

Arbeitman MN, Furlong EEM, Imam F, Johnson E, Null BH, Baker BS et al. (2002). Gene expression during the life-cycle of Drosophila melanogaster. Science 297: 2270-2275.

Brittnacher JG (1981). Genetic variation and genetic load due to the male reproductive component of fitness in Drosophila. Genetics 97: 719-730.

Chippindale AK, Gibson JR, Rice WR (2001). Negative genetic correlation for adult fitness between sexes reveals ontogenetic conflict in Drosophila. Proc Natl Acad Sci USA 98: 1671-1675.

Demuth JP, Wade MJ (2007). Maternal expression increases the rate of bicoid evolution by relaxing selective constraint. Genetica 129: 37-43.

Eanes WF, Hey J, Houle D (1985). Homozygous and hemizygous viability variation on the $\mathrm{X}$ chromosome of Drosophila melanogaster. Genetics 111: 831-844.

Fernández B, Garcia-Dorado A, Caballero A (2004). Analysis of the estimators of the average coefficient of dominance of deleterious mutations. Genetics 168: 1053-1069.

Frankham R (2005). Genetics and extinction. Biol Conserv 126 131-140.

Haag-Liautard C, Dorris M, Maside X, Macaskill S, Halligan D, Houle D et al. (2007). Direct estimation of per nucleotide and genomic deleterious mutation rates in Drosophila. Nature 445: 82-85.

Hoekstra HE, Hoekstra JM, Berrigan D, Vignieri SN, Hoang A, Hill CE et al. (2001). Strength and tempo of directional selection in the wild. Proc Natl Acad Sci USA 98: 9157-9160.

Innocenti P, Morrow EH (2010). The sexually antagonistic genes of Drosophila melanogaster. PLoS Biol 8: e1000335.

Ives PT (1970). Further genetic studies of the south Amherst population of Drosophila melanogaster. Evolution 24: 507-518.
Keller LF (1998). Inbreeding and its fitness effects in an insular population of song sparrows (Melospiza melodia). Evolution 52: 240-250.

Kodric-Brown A, Brown JH (1987). Anisogamy, sexual selection, and the evolution and maintenance of sex. Evol Ecol 1: 95-105.

Lynch M, Walsh B (1997). Genetics and analysis of quantitative traits. Sinauer: Massachusetts.

Mackay TFC (1985). A quantitative genetic analysis of fitness and its components in Drosophila melanogaster. Genet Res 47: 59-70.

Meagher S, Penn DJ, Potts WK (2000). Male-male competition magnifies inbreeding depression in wild house mice. Proc Natl Acad Sci USA 97: 3324-3329.

Miller PS, Hedrick PW (1993). Inbreeding and fitness in captive populations: lessons from Drosophila. Zoo Biol 12: 333-351.

Peng T, Moya A, Ayala F (1991). Two modes of balancing selection in Drosophila melanogaster: overcompensation and overdominance. Genetics 128: 381-391.

Pray LA, Goodnight CJ (1995). Genetic variation in inbreeding depression in the red flour beetle Tribolium castaneum. Evolution 49: 176-188.

Pusey AE (1987). Sex-biased dispersal and inbreeding avoidance in birds and mammals. Trends Ecol Evol 2: 295-299.

R Development Core Team (2010). R: A language and environment for statistical computing. R Foundation for Statistical Computing: Vienna, Austria. http://www.R-project.org.

Rice WR (1984). Sex chromosomes and the evolution of sexual dimorphism. Evolution 38: 735-742.

Rice WR (1996). Sexually antagonistic male adaptation triggered by experimental arrest of female evolution. Nature 381: 232-234.

Rose MR (1984). Laboratory evolution of postponed senescence in Drosophila melanogaster. Evolution 38: 1004-1010.

Rose MR, Charlesworth B (1981). Genetics of life history in Drosophila melanogaster. I. sib analysis of adult females. Genetics 97: 173-186.

Rowe L, Houle D (1996). The lek paradox and the capture of genetic variance by condition dependent traits. Proc Biol Sci 263: $1415-1421$.

Salathé M (2006). Sexual selection and its effect on the fixation of an asexual clone. Biol Lett 2: 536-538.

Siller S (2001). Sexual selection and the maintenance of sex. Nature 411: 689-692.

Slate J, Kruuk LE, Marshall TC, Pemberton JM, Clutton-Brock $\mathrm{TH}$ (2000). Inbreeding depression influences lifetime breeding success in a wild population of red deer (Cervus elaphus). Proc Biol Sci 267: 1657-1662.

Stolc V, Gauhar Z, Mason C, Halasz G, van Batenburg MF, Rifkin SA et al. (2004). A gene expression map for the euchromatic genome of Drosophila melanogaster. Science 306: 655-660.

Tomkins JL, Radwan J, Kotiaho JS, Tregenza T (2004). Genic capture and resolving the lek paradox. Trends Ecol Evol 19: 323-328.

Wedekind C (2002). Sexual selection and life-history decisions: implications for supportive breeding and the management of captive populations. Conserv Biol 16: 1204-1211.

Whitlock MC, Agrawal AF (2009). Purging the genome with sexual selection: Reducing mutation load through selection on males. Evolution 63: 569-582.

Willis JH (1999). Inbreeding load, average dominance and the mutation rate for mildly deleterious alleles in Mimulus guttatus. Genetics 153: 1885-1898.

Wilton AN, Sved JA (1979). X-chromosomal heterosis in Drosophila melanogaster. Genet Res 34: 303-315. 